\section{Ein Fall von Coma diabeticum, behandelt nach Stadelmann's Vorschlag.}

\section{Von Dr. A. Koettnitz, Zeitz.}

Dass das Coma diabeticnm zn jenen räthselhaften Vorgängen gehört, die als Antointoxicationen bezeichnet werden, darüber besteht wohl kanm noch ein Zweifel. Die Untersnchnngen von Stadelmann, Minkowski, Külz haben nahegelegt, dass es sich nm eine Sänreintoxication handelt, nm das Anftreten einer Sänre, die von Stadelmann als Crotonsäure, von Minkowski nnd Külz als Oxybuttersänre, die leicht bei chemischen Manipnlationen in erstere zerfällt, bestimmt wurde. Und mit dem Anftreten, mit dem Anwachsen dieser Sănre im Harn droht Coma! (Nannyn ${ }^{1}$ ).

Stadelmann hatte in Rücksicht auf diesen Befnnd - dem Anftreten von Aceton nnd Acetessigsänre spricht er keine grosse Bedentnng zn - einen Vorschlag znr Behandlnng dieser absolnt nngünstigen Anfälle gemacht, nud zwar dahin, möglichst grosse Dosen von Alkalien znr Paralysirnng dieser Sänre dem Körper einznverleiben, speciell intravenöse lnjectionen 3-5\% Lösungen von kohlensanrem Natron vorznnebmen.

Die Erfahrnngen, die man bis jetzt mit dieser Behandlnng gemacht, sind leider nicht als günstige zn bezeichnen. Von 11 Fällen, die Stadelmann ${ }^{2}$ ) ans der Litteratnr znsammenstellen konnte, ist nnr einer in Genesung übergegangen. Der Antor rügt nnn, dass in einigen Fällen (einen scheidet er ïbrigens als nicht hierhergehörig wohl mit Recht ans) von seiner Vorschrift abgewichen nnd statt kohlensauren Natrons doppeltkohlensanres in Anwendung gebracht worden ist.

Er rügt diese Abweichung als nuzweckmässig, da das doppeltkohlensanre Natron überschüssige Kohlensäure enthält". Nnn hat das Blnt jener Diabetiker an Alkalescenz verloren, es kann selbst nentral saner reagiren, so dass freie Säuren oder sanre Salze vorhanden sein müssen, welche ans einer Lösnng von doppeltkohlensanrem Natron die Kohlensäure als freies Gas mit Leichtigkeit anstreiben."

Von dieser freien Kohlensänre kann er keinen Nntzen sehen. „Der Kohlensänregehalt des Blntes ist bei den Diabetikern im Coma ein nngemein niedriger, nnd dieses beweist nach nnseren Erfahrnngen, dass die fixen Alkalien des Blutes dnrch andere Sänren in Beschlag genommen sind, nnd dass folglich die Gewebe, ans welchen das Blnt die Kohlensänre abfïhren soll, mit derselben ïberladen sind." "Und wer weiss, einen wie grossen Antheil an dem Bilde des Coma diabeticnm diese Ueberladnng des Körpers mit Kohlensänre hat."

Anf Grund theoretischer Erwägnngen nud der Ergebnisse von Thierversnchen empfiehlt er, als Grnndlage der Injection die physiologische Kochsalzlösnng und anf $150 \mathrm{ccm}$ der Fln̈ssigkeit $7,2 \mathrm{~g}$ $\mathrm{Na}_{2} \mathrm{CO}_{3}$ nnd 4,6 g NaHCO 3 zn nehmen. Von dieser Lösung, die übrigens anch schwächer sein kann, soll man nnter genaner Controlle von Puls, Athmnng etc. so viel injiciren, dass es gelingt, den Urin alkalisch zn machen.

In einem Falle hatte ich nnn Gelegenheit, nach Stadelmann's Vorschlag verfahren zn können. Trotzdem der Fall letal ablief, trotzdem er nnr sehr lückenhaft beobachtet werden konnte, halte ich ihn doch für interessant genug, ihn knrz mitzntheilen.

Fran L. in Zeitz, 43 Jahre alt, leidet seit etwa 3 Jahren an Diabetes. Sie hatte 6 Schrangerschaften durcbgemacht, aber nur zwei Kinder ansgetragen, davon ist das eine Idiot. Sie ist immer nervös gewesen; vor etra drei Jabren traten zn diesen Beschwerden nocb Polynrie nnd Polydipsie hinzn, und diese Umstănde führten zur Entdeckung des Diabetes, der vielleicbt scbon länger bestanden hatte.

Im letzten Jahre, wo ich Patientin in Bebandlnng hatte, schwankte der Zuckergehalt des Urins zwisehen $31 / 2-4 \%$, manchmal erreicbte er die Höhe von $70 \%$. Patientin, die vor etwa $2 \frac{1}{2}$ Jahren nnerwartet den Gatten (an Gehirnapoplexie) verloren und seit dieser Zeit noch nervöser geworden, zeigte bei ihrem Eintritt in meine Behandlnng das Bild der schwersten Neurastbenie. Sie war zn geistiger wie körperlicher Arbeit absolnt untauglich, sie war aller Energie bar, dabei stets voller Klagen über Kopfweh, n̈ber Reissen in den verscbiedensten Nerven, znmal in den Iscbiadicis. Der Urin enthielt oft Aceton.

Ansser diesen nervösen Bescbwerden litt sie oft an Lnftröhrenkatarrh, später noch an Vnlvo-Vaginitis mit Furnnkelbildung.

Patientin hatte schon alle möglichen Mittel genommen, alles brachte wenig Linderung. Anch ich versuchte verschiedenes, am besten wirkte schliesslich ein mehrwöchentlicher Landanfenthalt bei mässig strenger Diät

1) Nannyn, Die diaetetische Bebandlnng des Diabetes mellitus. Volkmann's Sammlnng klin. Vorträge No. $349 / 350$ p. 18

2) Stadelmann, Klinisches und Experimentelles n̈ber Coma diabeticnm nnd seine Behandlnng. Dentsche med. Wochenscbrift 1889 No. 46. nnd mässiger Bewegnng. Als Medication wnrden Pillen von Lithion salicylicnm nnd Lithion benzoicum genommen.

Die Bessernng bielt den ganzen Herbst (1889) an. Am 31. December trat nun die Katastropbe ganz nnerwartet ein.

Früh gegen 1 Uhr bekam Patientin Unrnhe, Angstanfälle; da die Grippe regirte, hatte sie grosse Furcht, dass sie davon befallen wäre. Danı stellten sich heftige Kopfschmerzen ein, dazn Ziehen in den Gliedern, F'rösteln nnd Erbrecben. Friih 5 Uhr kann sie nicht gehen, ,sie hatte es in den Beinen"; dabei fröstelt nnd friert sie weiter. Gegen $8 \mathrm{Ubr}$ wird sie benommen, gegen 9 Uhr verfällt sie in Schlaf.

Um $12 \mathrm{Uhr}$ finde ich Patientin bewnsstlos, von blaurothem Aussehen, mit kalten Händen, schnellem nnd schwachem Puls, ohne Fieber, in tiefstem Coma.

Erst $21 / 2$ Uhr nachmittags kam ich dazu (es gab eine Unzahl Grippekranker), die Infusion vorznnehmen. Anf 1 Liter warmer physiologischer Kochsalzlösung, die mit einem Tropfen Natronlange alkaliscb gemacbt, wurden $30,0 \mathrm{~g}$ Natr. bicarb. nnd $42,0 \mathrm{~g}$ Natr. carb. pnr. gelöst. Die Infusion wurde in eine Vene der linken Elllbogenbenge gemacbt; das anssickernde Blnt hatte n̈brigens eine merkwürdig brauırothe Farbe.

Nach Eingiessung von ea. $1 / 2$ Liter der Flüssigk eit trat eine anffallende Aenderung in dem Znstande der Kranken ein. Die Patientin wird warm; sie fängt an, sich wieder zn bewegen; sie giebt wieder Antworten, allerdings ohne die Augen zu öffnen.

Die Infusion wurde sistirt; alles aber, anch nene Flüssigkeit, zur Wiederholung bereitgestellt.

Leider mnsste ich inzwischen Patientin verlassen; als ich abends $7^{1} / 2$ Ubr wieder eintraf, war Patientin eben verschieden. Die Bessernng liatte nur knrze Zeit, kanm 10-15 Vinuten angebalten, dann war der alte Znstand wiedlergekehrt.

So lückenhaft nnn anch nnser Fall beobachtet worden ist, so nnvollkommen auch die Behandlung dnrch die Ungunst der Verhältnisse sich gestaltet hat, immerhin schien mir die Pnblication des Falles angezeigt und nicht ohne Interesse zn sein.

Man könnte wohl Zweifel hegen, dass es sich nm echtes Coma diabeticum gehandelt, znmal da keine Urinnntersnchnng stattgefnnden hat. Doch wüsste ich nicht, wie man den Znstand anders denten wollte. Man könute zwar an Grippe denken, znmal wenn man die verderbliche Wirknng, die sie entfaltete, wenn sie zn anderen Affectionen hinzntrat, ins Ange fasst.

So verlor ich einen leichtkranken Phthisiker, der an Grippe mit einseitiger Plenritis exsudativa nnd doppelseitiger Bronchitis erkrankte, in wenigen 'Tagen; einen Mann mit linksseitiger Lnngenschrumpfung (Folge überstandener eitriger Rippenfellentziindnng) an Grippe mit rechtsseitiger Pnenmonie in zwei Tagen; eine Gravida in sechsten Monat an Grippe mit Pnenmonie, nachdem Frühgeburt eingetreten, innerhalb fünf Tagen; eine Diabetica mit Strnma (Consultationsfall) starb unerwartet, ohne überhaupt bettlägerig gewesen zu sein, innerhalb zweier Stnnden, nachdem sie fünf Tage znvor von leichter Grippe ohue jede Complication befallen worden war.

Aber all diese Fälle hatten doch 'Tage lang zngebracht, hatten fast alle Grippe mit schweren Complicationen! Unsere Patientin starb in kanm 18 Stnnden; nnd all die Erscheinnngen, die sie darbot, wie Frösteln, Ziehen in den Gliedern, Angstanfälle, Kopfschmerzen waren bei ihr gewöhnliche Vorkommnisse. Immerhin ist die Möglichkeit, dass Grippe mit im Spiele gewesen, nicht ganz von der Hand zn weisen.

Interessant nnd in die Angen springend war die Aendernng, die sich während der Infnsion in dem Znstande der Kranken bemerkbar machte.

Ich muss gestehen, dass ich sehr skeptisch an diesen therapentischen Eingriff heranging. Um so mehr war ich erstannt über die anffallende Bessernng. Die Patientin, die noch eben im tiefsten Coma ohne jede Reaction mit kalten Gliedern dagelegen hat, fängt plötzlich an, sich zn bewegen nud zn strecken, Schweiss zeigt sich auf der Stirn, die Sprache nnd das Bewnsstsein kommen wieder: die Patientin giebt Antworten, ja erkennt ihre Ungebnng an der Stimme.

Dass schliesslich doch Exitns eingetreten, das ist dem Stadelmann'schen Verfahren nicht znm Vorwnrf zn machen. Die ganze Behandlnng nnd Beobachtnng war dnrch die Ungunst der Verhältnisse eine so lückenhafte, die Kranke kam so spät znr Infusion, dass der Tod nur natürlich erscheinen kann.

Immerhin könnte man einige theoretische Erwägungen nnd Bedenken gegen das Verfahren vorbringen.

Angenommen, dass wirklich eine Sänreintoxication (dnrch Bildnng von Oxybnttersänre) vorliegt - nnd die Entdecknng Fichtner's'), der die Epithelien der gewnndenen Harncanälchen bei solchen Fällen von Coma fettig degenerirt mit eigenthümlicher Anordnnng der Fetttröpfchen an der Peripherie des Canals fand, würde nicht dagegen sprechen, da die Verändernngen wohl durch die Sänreintoxication vernrsacht sein könnten - was erreichen wir dnrch die Infusion des kohlensanren Natrons?

1) cfr. Jahresbericht von Virchow und Hirsch 1890 Bd. II Abtheil. I 
Wir wollen die Säure paralysiren durch ein für das Blut indifferentes Salz; wir wissen aber nicht, ob das neugebildete Salz ohne Bedeutung für das Blut und den Körper ist. Und dann weiter, das Blut ist das Medium, in dem sich der chemische Vorgang der Bindung der Oxybuttersäure durch kohlensaures und doppeltkohlensaures Natron abspielen, in dem die neue chemische Verbindung auftreten soll; nicht einfaches Wasser ist es, wo der chemische Vorgang in Scene tritt, nein, das Blut, dieses Conglomerat von chemischen Verbindungen und Constitutionen. Wird hierin der Vorgang sich so einfach abspielen, ohne weitere Kreise zu ziehen, ohne gleichzeitig andere chemische Umsetzungen zu veranlassen? Bei jedem chemischen Vorgange wird Wärme frei, in unserem Falle auch gleichzeitig Kohlensäure entwickelt. Sollten diese Momente, zumal das letztere, keine Bedeutung haben? Die Kohlensäure befindet sich in statu nascendi, sie ist überhaupt kein gleichgültiger Factor im Blut und in den Geweben. Wissen wir doch nicht, wie Stadelmann selbst betont, einen wie grossen Einfluss die Kohlensäure beim Zustandekommen des Comas ausübt.

Alles dies sind aber nur theoretische Erörterungen und Erwägungen, die hinfällig werden, wenn die klinischen Erfahrungen zu Gunsten der Infusion sprechen. Auffallend bleibt entschieden die in unserem, wie in anderen Fällen bewirkte Besserung des $\mathrm{Zu}$ standes, so vorübergehend sie auch war.

Allerdings lässt sich fragen, kann man nicht auf eine andere Weise, die weniger eingreifend ist, dasselbe erreichen?

Sabli ${ }^{1}$ ) hat in einer interessanten Arbeit auf die Wirkung subcutaner Injectionen physiologischer Kochsalzlösungen aufmerksam gemacht und sie unter anderem als ein werthvolles Mittel bei Bekämpfung schwerer toxischer Zustände empfohlen.

Man erreicht dadurch in erster Linie eine Diluirung des im Blute kreisenden Giftes und dann durch die sehr oft eintretende Erböhung der Diurese eine "Auswaschung "des mensch lichen Körpers. Gestützt auf Versuche an Kranken, z. B. Fällen mit Status typhosus, empfiehlt er diese Injectionen bei den Zuständen, die als Autointoxicationen bezeichnet werden, speciell beim Coma diabeticum, „bei dem trotz aller theoretischen Erwartungen die alkalischen Eingiessungen bekanntlich wenig leisten".

$\mathrm{Ob}$ in unserem Falle die augenscheinliche Besserung nur als das Resultat einer Diluirung des vorhandenen Giftes oder als Folge einer specifischen Wirkung der Injectionsflüssigkeit aufzufassen ist, das wage ich nicht zu entscheiden. Ich habe die Sahli'sche Methode bei mehreren sehr schweren und stets letal endenden Fällen in Anwendung gezogen; die Wirkung war stets eine auffallende Besserung des Zustandes. Bei einem Falle von Perforationsperitonitis (Folge von Typhlitis und Perityphlitis) war die Wirkung unverkennbar; der Puls hob sich, die Glieder wurden warm, Schweiss trat ein. Ebenso günstig zeigte sich die Wirkung bei einem Falle von Ileus und einem Falle von Cholera infantum. Freilich beruhte hier die günstige Wirkung nicht allein in einer Verdüunung des Giftes, als vor allem in der Aufhebung der Wasserverarmung der Gewebe.

Ob nun die Sahli'schen subcutanen Injectionen, die leicht, bequem und ungefährlich sind, dasselbe oder noch mehr leisten als die Stadelmann'schen intravenösen Infusionen in der Bekämpfung des Coma diabeticum, das muss erst die Zukunft lehren.

Jedenfalls verdienen die Sahli'schen Injectionen bei ähnlichen Zuständen, ich nenne in erster Linie die Eklampsie der Kreissenden, in Anwendung gezogen zu werden. 